\title{
APPROCHE MULTIDISCIPLINAIRE D’UNE SÉQUENCE LACUSTRE HOLOCÈNE DANS LES ALPES DU SUD AU LAC PETIT (MERCANTOUR, ALT. 2200 M, FRANCE): HISTOIRE D’UN GÉOSYSTÈME DÉGRADÉ
}

\author{
Elodie BRISSET ${ }^{1,2}$, Frédéric GUITER ${ }^{1}$, Cécile MIRAMONT ${ }^{1}$, Claire DELHON ${ }^{3}$, \\ Fabien ARNAUD ${ }^{4}$, Jean-Robert DISNAR ${ }^{5}$, Jérôme POULENARD ${ }^{4}$, \\ Edward ANTHONY ${ }^{2}$, Jean-Dominique MEUNIER ${ }^{2}$, Bruno WILHELM ${ }^{4}$ \\ \& Christine PAILLES ${ }^{2}$
}

\begin{abstract}
RÉSUMÉ
L'analyse multiproxies à haute résolution d'une séquence sédimentaire prélevée dans le Lac Petit (alt. $2200 \mathrm{~m}$, vallon des Millefonts, Mercantour, France) a permis d'étudier les interactions climat-environnement-Homme à l'Holocène récent en contexte de haute montagne dans les Alpes du Sud. Grâce aux analyses lithostratigraphiques, géochimiques et palynologiques, trois grandes phases ont pu être reconstituées: (1) une période de stabilité relative de l'écosystème de 4750 à 4350 cal. BP, caractérisée par une sédimentation lacustre dominante et des sols développés sur les versants, (2) de 4350 à 1550 cal. BP, une augmentation des apports érosifs contemporaine d'une dégradation des sols superficiels et du couvert forestier, (3) une phase de déstabilisation intense et définitive des versants depuis $1550 \mathrm{cal}$. BP. Le climat a certainement joué un rôle dans la dynamique du bassin-versant, mais l'Homme, dont l'impact reste discret jusqu'à $1550 \mathrm{cal}$. BP, pourrait être un facteur majeur de perturbation des écosystèmes du vallon des Millefonts pendant le Subatlantique. Enfin, la récurrence d'apports terrigènes pourrait témoigner d'évènements orageux de forte intensité dont les conséquences érosives ont pu être amplifiées localement par l'augmentation de la pression agro-pastorale et des activités minières.
\end{abstract}

Mots-clés: sédiment lacustre, érosion des sols, anthropisation, végétation, pollen, Holocène, Alpes du Sud, évènement de 4200 cal. BP

\section{ABSTRACT}

A MULTIDISCIPLINARY INVESTIGATION OF A HOLOCENE LAKE SEDIMENT SEQUENCE IN THE SOUTHERN FRENCH ALPS AT LAKE PETIT (MERCANTOUR, 2200 M A.S.L., FRANCE): HISTORY OF A DISTURBED GEOSYSTEM High resolution multiproxy analyses, including sedimentological, geochemical and palynological investigations, were carried out on a 144-cm-long sediment profile from Lake Petit (2200 m a.s.1., Vallon des Millefonts, Mercantour, in the French Southern Alps). This allowed us to reconstruct complex interactions between climate, environment and human during the Late Holocene. We identify three phases: (1) a first phase (4750 to $4350 \mathrm{cal}$. BP) characterized by a dominant lake sedimentation and developed soils on slopes, (2) a second period (4350 to $1550 \mathrm{cal}$. BP) marked by an abrupt switch in sediment source and an input of organic allochthonous matter linked with soil erosion and a more open landscape, (3) a final phase (since 1550 cal. BP) of intense destabilization of slopes. Even if climate could have triggered such drastic changes, discreet but continuous human impact until 1550 cal. BP might be the main factor of degradation of slopes and ecosystems in the valley. The record of abundant terrigenous input also suggests that high-intensity rainfall events might have occurred, while agro-pastoralism and local mining activities continuously weakened slopes.

Keywords: lacustrine sediment, soil erosion, human impact, vegetation, pollen, Holocene, French Southern Alps, 4.2 ka event

\footnotetext{
${ }^{1}$ Institut Méditerranéen de Biodiversité et d'Ecologie (IMBE), Aix-Marseille Université, CNRS. Europôle Méditerranéen de l'Arbois, bât. Villemin, BP80, F-13545 AIX-EN-PROVENCE cedex 04.Courriel : brisset@cerege.fr

${ }^{2}$ Centre Européen de Recherche et d'Enseignement des Géosciences de l'Environnement (CEREGE), Aix-Marseille Université, CNRS. Europôle Méditerranéen de l'Arbois, BP 80, F-13545 AIX-EN-PROVENCE cedex 04.

3 Centre d'Etudes Préhistoire, Antiquité, Moyen Âge (CEPAM), Université de Nice, CNRS. Campus Saint-Jean-d'Angély, SJA3, 24 avenue des Diables Bleus, F-06357 NICE cedex 04.

${ }^{4}$ Laboratoire Environnement Dynamique et Territoire de Montagne (EDYTEM), Université de Savoie, CNRS. Pôle Montagne, Campus scientifique, F-73376 LE-BOURGET-DU-LAC cedex.

${ }^{5}$ Institut des Sciences de la Terre d'Orléans (ISTO), Université d'Orléans, CNRS. Bâtiment des Géosciences, rue de St-Amand, BP 6759, F-45067 ORLÉANS cedex 02 .
} 


\section{1 - INTRODUCTION}

Les milieux de montagne sont des géosystèmes fragiles soumis à d'importants gradients géomorphologiques, climatiques et écologiques. Tout changement environnemental - qu'il soit d'origine naturelle ou anthropique peut déstabiliser profondément ces milieux (Demangeot, 1984). La reconstitution de l'histoire paléoenvironnementale de ces zones de montagnes représente un enjeu d'importance dans la compréhension des mécanismes de résilience de ces géosystèmes.

Les paysages de montagne des Alpes du Sud sont affectés depuis plusieurs millénaires par l'activité humaine (e.g. Nicod, 2008). Les premières traces ténues d'incursion humaine en moyenne et haute altitude dans les Alpes méridionales datent du Paléolithique final (Mocci et al., 2005). Plus significatives sont les récentes découvertes de sites du Mésolithique dans l'Argentiérois et l'Ubaye (Garcia et al., 2007). Au Néolithique les traces de fréquentation humaine se font plus fréquentes (enclos pastoraux, haltes de chasse); puis c'est à partir du Néolithique final et de l'âge du Bronze que la documentation archéologique révèle une présence humaine bien implantée (Mocci et al., 2008a). Les études couplant des données archéologiques et paléoécologiques (palynologie et anthracologie) montrent, à partir d'environ 7000 cal. BP, l'impact des pratiques agro-pastorales sur les environnements végétaux de montagne avec des périodes successives de pression ou de déprise des sociétés sur les territoires (Court-Picon, 2005; Mocci et al., 2008a, b). Enfin, des traces d'activités minières (extraction et réduction) - pratiques qui affectent profondément le couvert végétal et les dynamiques érosives et sédimentaires -ont été découvertes en altitude dans les Alpes du Sud et apparaissent dès l'âge du Bronze (Rostan \& Rossi, 2002; Bourgarit et al., 2008).

Le vallon des Millefonts (commune de Valdeblore, Alpes-Maritimes, fig. 1A) constitue une zone privilégiée pour aborder la délicate problématique des interactions entre le climat, l'environnement montagnard et l'Homme à l'Holocène. En effet, le vallon est occupé par une série de dépressions lacustres qui se comportent comme des pièges naturels à sédiments et permettent d'enregistrer la variabilité paléoenvironnementale à long terme. Les investigations archéologiques qui ont eu lieu dans le vallon (Ancel, 2010; Morin \& Rosenthal, 2003; Suméra, 2007; Suméra et al., 2008; Pagès, 2009) ont notamment montré qu'une activité d'extraction et de réduction de minerai de fer y a pris place au cours du Moyen Âge. Dans le cadre du Projet Collectif de Recherche intitulé «Peuplement et occupation du sol du Massif du Mercantour et de l'Argentera au cours de l'Holocène» (coordination F. Suméra, Centre Camille Jullian), un profil sédimentaire a été prélevé dans la dépression lacustre principale du vallon (Lac Petit). Le but de cet article est de présenter les recherches paléoenvironnementales en cours visant à (1) reconstruire l'évolution paléoenvironnementale d'un bassin-versant de montagne péri-méditerranéenne et (2) retracer l'impact et les interactions des différents forçages (naturels et anthropiques) sur cette évolution.

\section{2 - SITE D'ÉTUDE : LE LAC PETIT}

Le vallon des Millefonts se situe sur la terminaison sud des Alpes françaises (fig. 1A) dans le massif du Mercantour. Le Lac Petit (2200 m) est le plus à l'aval d'un chapelet de 5 lacs de surcreusement glaciaire connectés entre eux par des écoulements intermittents. La surface du lac est gelée en saison hivernale (novembre à mars). Le niveau d'eau du lac Petit a été rehaussé d'1,5 mètre suite à la construction d'un barrage en 1947. Ses dimensions actuelles sont de $160 \mathrm{~m}$ de longueur pour $140 \mathrm{~m}$ de large avec une profondeur maximale de $7 \mathrm{~m}$. Le lac draine un bassin-versant de $2,5 \mathrm{~km}^{2}$ caractérisé par des affleurements du substratum métamorphique (gneiss et migmatites). Son régime hydrologique est dominé par la fonte des neiges printanières et estivales. Bien que d'exposition sud, la limite supérieure de la forêt se situe en deçà de la cuvette lacustre vers $2100 \mathrm{~m}$. Autour du lac, les versants sont couverts par des pelouses alpines à Ericaceae (fig. 1B).

\section{3 - MATÉRIEL ET MÉTHODES}

Le profil sédimentaire PET09P2 (144 cm) a été prélevé en juin 2009 avec un carottier gravitaire UWITEC. Après ouverture au laboratoire, la carotte a été décrite et photographiée. La distribution de la taille des particules a été mesurée par granulométrie laser (Malvern Mastersizer S) tous les $5 \mathrm{~mm}$ après une perte au feu de la matière organique à $550^{\circ} \mathrm{C}$.

Les éléments $\mathrm{Si}, \mathrm{Ti}$, et $\mathrm{K}$ ont été mesurés par fluorescence de rayons-X sur un Itrax Core Scanner selon un pas de $2 \mathrm{~mm}$ (source des RX: molybdène, temps de comptage de 60 secondes). Cette méthode mesure les variations d'abondance des éléments le long de la séquence sédimentaire (Croudace et al., 2006). Les éléments $\mathrm{K}$ et Ti co-varient en fonction de la teneur en argiles dans le sédiment. Nous utiliserons le rapport Ti/Si comme traceur du détritisme, la normalisation permettant de mettre en valeur des effets de dilution.

Le Carbone Organique Total (\%COT) et les Indices d'Hydrogène (IH) et d'Oxygène (IO) ont été mesurés de façon ponctuelle sur 25 échantillons par pyrolyse RockEval (Espitalié et al., 1985). L'indice d'Hydrogène (mg $\mathrm{HC} / \mathrm{g}$ COT) mesure la quantité d'hydrocarbures émis lors de la pyrolyse rapportée à la teneur en Carbone Organique Total (COT). L'indice d'Oxygène quantifie l'émission du dioxyde de carbone rapporté au \% COT ( $\left.\mathrm{mg} \mathrm{CO}_{2} / \mathrm{g} \mathrm{COT}\right)$. Ces indices permettent de caractériser la qualité et l'origine de la matière organique selon son degré d'évolution ou de dégradation.

L'abondance en silice biogénique (\%BSi) a été analysée sur 25 échantillons selon la méthode de digestion alcaline à chaud (DeMaster, 1981). La concentration en silice dissoute a été mesurée par spectrocolorimétrie en réaction au jaune de molybdène sur un Jasco V-650 Spectrophotometer.

Les abondances relatives en silice biogénique et en matière organique ont été déterminées par spectroscopie 

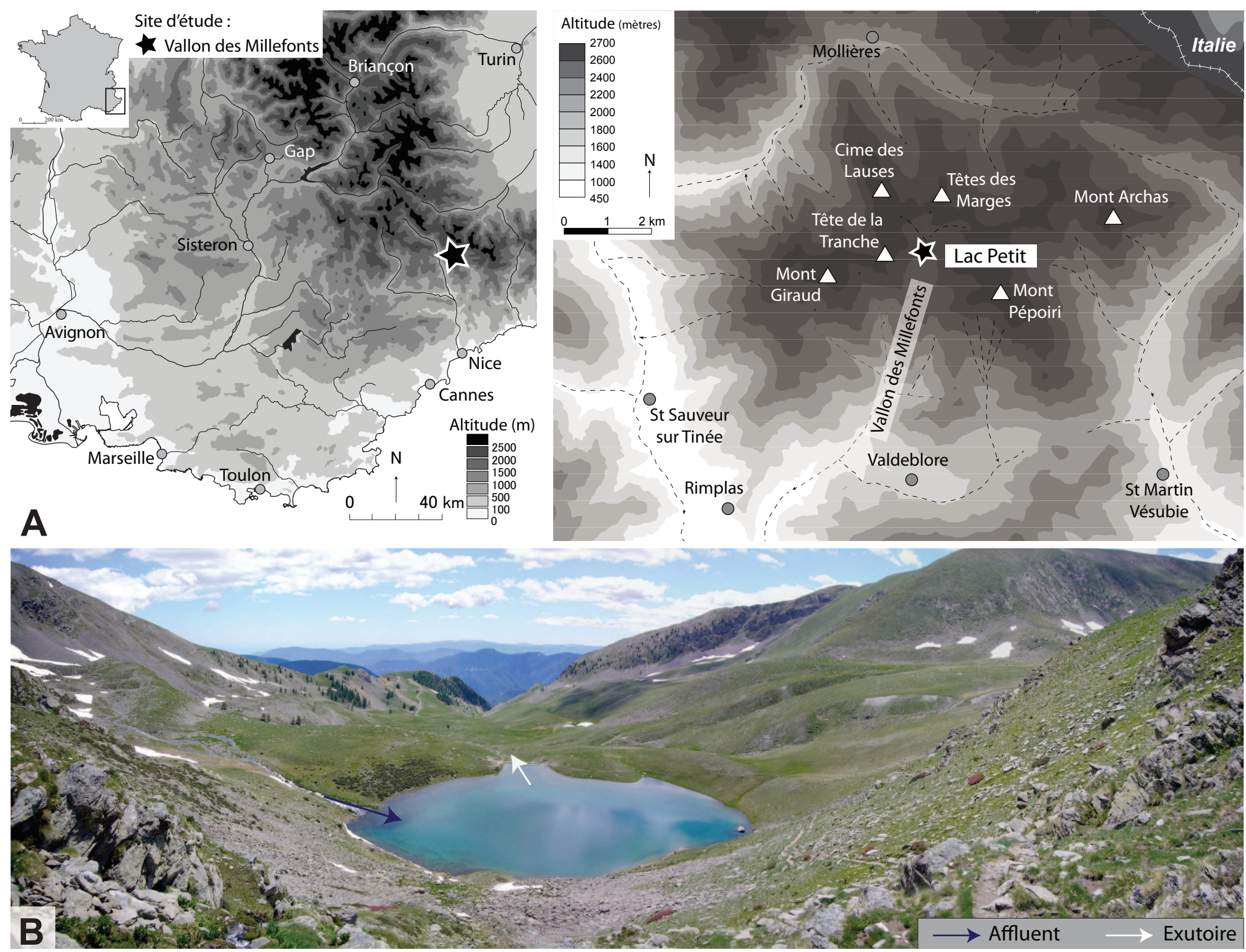

Fig. 1: (A) Localisation du site d'étude, (B) Panorama du bassin-versant de Millefonts. Le lac Petit est bordé d'une pelouse alpine à éricacées. Un bosquet de mélèze visible en arrière plan constitue l'actuelle limite supérieure des arbres à $2100 \mathrm{~m}$ d'altitude.

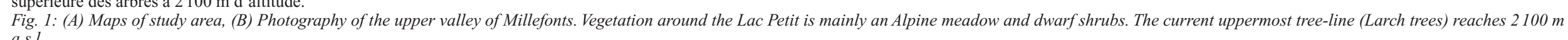
a.s.l. 
infrarouge à transformée de Fourier (IRFT) NICOLET 380 (Smart Diffuse Reflectance). Dans cette étude, la méthode IRFT est utilisée en complément aux méthodes largement employées pour la quantification de la silice biogénique et du carbone organique total (Rosén et al., 2010). Le gain de temps analytique réalisé par cette méthode nous a permis de travailler en haute résolution d'échantillonnage (échantillonnage en continu tous les $1 \mathrm{~cm}$ ). Deux modèles de régression PLS (Partial Least Square) ont été calculé entre les spectres infrarouges et d'une part, les mesures en $\% B S i\left(n=25, r^{2}=0,80\right.$, erreur résiduelle $=5,99 \%, \mathrm{p}<0,01)$, et d'autre part les mesures en $\%$ TOC $\left(n=24, r^{2}=0,94\right.$, erreur résiduelle $=0,62 \%$, $\mathrm{p}<0,01)$. Ces modèles PLS ont été utilisés pour prédire les concentrations en \%BSi et \%TOC des 144 échantillons mesurés seulement avec la méthode infrarouge.

Une analyse pollinique préliminaire a été réalisée sur 59 échantillons prélevés à volume constant selon une résolution moyenne de $3 \mathrm{~cm}$. La méthode d'extraction du matériel sporo-pollinique fossile utilisée est décrite dans Faegri et Iversen (1989). A ce stade de l'étude, les premiers résultats sont présentés sous la forme de courbes synthétiques:

- des taxons d'anthropisation (regroupant les fréquences polliniques de Plantago lanceolata, Plantago major, Plantago coronopus, Mentha, Rumex, Urtica, et des Chenopodiaceae);

- du rapport PA/PNA (pollen arboréen / pollen non arboréen). Cette courbe retrace la dynamique forestière et le degré d'ouverture des paysages;

- des taxons de la chênaie mixte (Quercus caduc., Tilia, Acer, Ulmus);

- des taxons de la hêtraie-sapinière (Abies, Fagus).

Les courbes du noyer (Juglans), dont la culture s'est généralisée en Europe à l'époque romaine et du pin (Pinus) sont également représentées dans le diagramme synthétique. Enfin, les stomates et trachéides de résineux ont été dénombrés, afin de préciser la présence ou l'absence de conifères (notamment du pin) aux abords du lac.

Sept datations ${ }^{14} \mathrm{C}$ AMS sur fragments de bois et débris végétaux terrestres déterminés spécifiquement ont été obtenues sur la carotte PET09P2 (tab. 1). Le modèle âge-profondeur a été calculé selon une interpolation spline paramétrée avec un ajustement semi-contraint aux fonctions de densité de probabilité (2 écarts-type) des 7 datations ${ }^{14} \mathrm{C}$ (module MCAgeDepth 0.1, Higuera et al., 2009).

\section{4 - RÉSULTATS}

\section{1 - CHRONOLOGIE}

L'enregistrement sédimentaire couvre les 4750 dernières années ( \pm 200 ans) (fig. 2). Le taux de sédimentation moyen est de $0,7 \mathrm{~mm} / \mathrm{an}$. Ce taux d'accumulation, relativement lent, est caractéristique d'une faible contribution des apports terrigènes à la sédimentation totale.

\section{2 - LITHOSTRATIGRAPHIE}

La carotte sédimentaire (fig. 3) est constituée d'une diatomite jaune-foncé/vert à texture grumeleuse piquetée

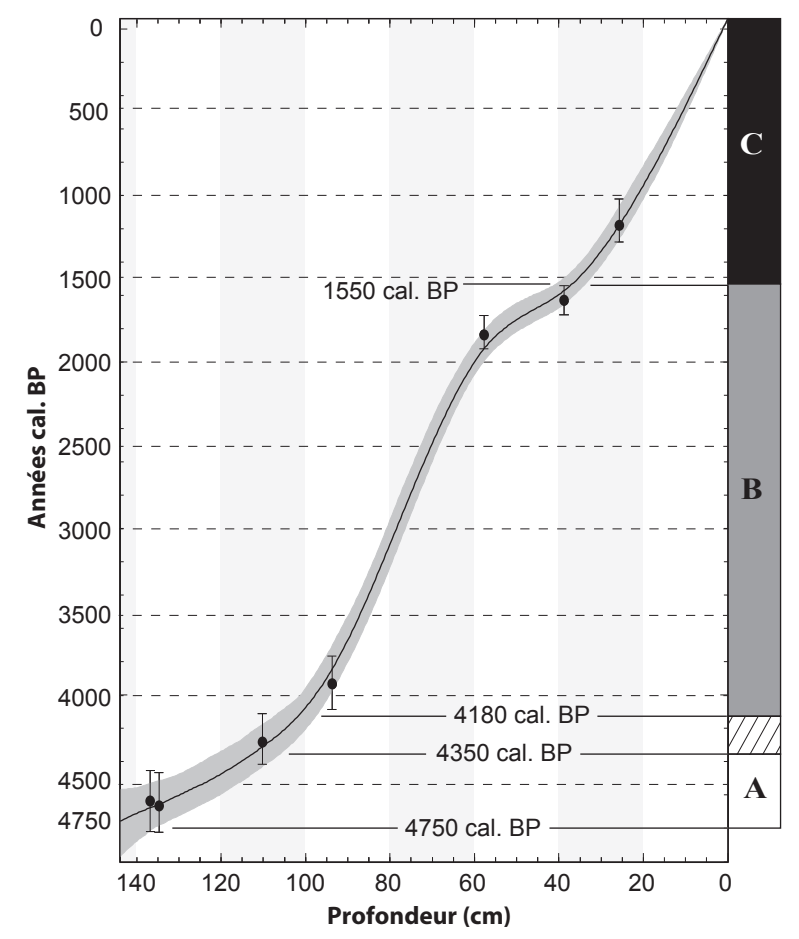

Fig. 2 : Relation âge-profondeur de la carotte PET09P2 (noir). L'intervalle de probabilité du modèle est représenté à 2 sigmas (enveloppe grisée).

Fig. 2: Age-depth model (black line) of PET09P2 and the 2 sigmas probability envelope (grey).

\begin{tabular}{|c|c|c|c|c|c|}
\hline $\begin{array}{c}\text { Profondeur } \\
(\mathbf{c m})\end{array}$ & $\begin{array}{c}\text { Code du } \\
\text { laboratoire }\end{array}$ & $\begin{array}{c}\text { Age }^{14} \mathrm{C} \\
\text { BP }\end{array}$ & $\begin{array}{c}\text { Age calibré à } 2 \sigma \\
(\text { cal. BP) }\end{array}$ & $\begin{array}{c}\text { Age calibré à 2 } \sigma \\
\text { (cal. AD / BC) }\end{array}$ & Matériel daté \\
\hline 26 & Poz-32578 & $1240 \pm 40$ & $1068(1179) 1270$ & $680 \mathrm{AD}(772 \mathrm{AD}) 882 \mathrm{AD}$ & Débris végétaux terrestres \\
\hline 39 & Poz-39213 & $1720 \pm 30$ & 1554 (1629) 1704 & 246 AD (322 AD) 396 AD & Bois - Larix \\
\hline 58 & Poz-35509 & $1890 \pm 30$ & 1733 (1840)1894 & 56 AD (111 AD) 217 AD & Bois de conifère \\
\hline 94 & Poz-32576 & $3620 \pm 40$ & 3835 (3932) 4080 & 2131 BC (1982 BC) 1886 BC & Débris végétaux terrestres \\
\hline 111 & Poz-35507 & $3855 \pm 35$ & 4155 (4281) 4411 & 2462 BC (2331 BC) 2206 BC & Ecaille de conifère \\
\hline 135 & Poz-39212 & $4125 \pm 35$ & 4528 (4666) 4820 & $2871 \mathrm{BC}(2716$ BC) $2579 \mathrm{BC}$ & Brindille - Ericaceae \\
\hline 137 & Poz-32577 & $4110 \pm 35$ & 4455 (4636) 4818 & 2869 BC (2686 BC) 2506 BC & Débris végétaux terrestres \\
\hline
\end{tabular}

Tab. 1 : Datations AMS ${ }^{14} \mathrm{C}$ réalisées sur la carotte PET09P2 du lac Petit.

Les datations ont été calibrées avec le logiciel Calib 6.0.2 utilisant la courbe de calibration IntCa109 (Reimer et al., 2009). Les âges calibrés sont donnés de la manière suivante [borne minimale à 2 sigmas; âge de la probabilité maximum; borne maximale à 2 sigmas].

Tab. 1: ${ }^{14}$ C AMS dates of core PET09P2 of Lake Petit. The calibration was performed using Calib 6.0.2, using the calibration curve IntCal09 (Reimer et al., 2009). Calibrated ${ }^{14} \mathrm{C}$ ages are presented with [2 sigmas range minimum; age of the maximal probability; 2 sigmas range maximum]. 


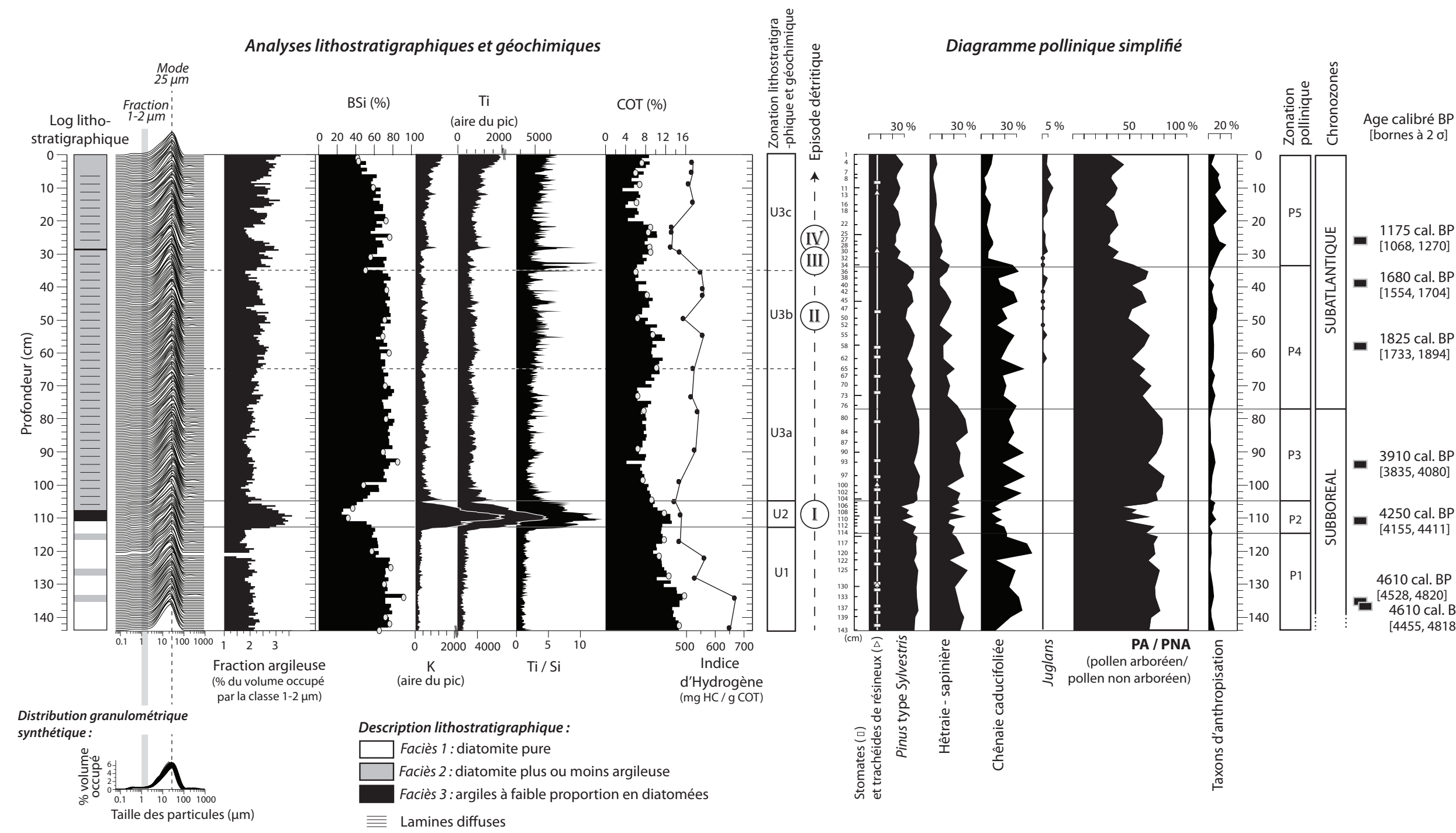

Fig. 3 : Analyses granulométriques, géochimiques et diagramme pollinique simplifié de l'archive lacustre PET09P2.

Pour les resultats en \%BSi et \%COT, les courbes noires pleines correspondent aux données infrarouges calibrées par les modèles de régression PLS. Les dosages quantitatifs en \%BSi et \%COT sont représentées par des points gris. Fig. 3: Grain size parameters, geochemical analysis, and simplified pollen diagram of core PETO9P2. The \%TOC and \%BSi PLS-regression models of IRFT measurements are represented by a black curve. The discrete quantitative measurements of \%TOC and \%BSi are shown by grey dots. 
de débris organiques (faciès 1 ) et un sédiment riche en diatomées plus ou moins argileux brun-jaune à brunmoyen (faciès 2). Le faciès 3 est caractérisé par un matériel argileux brun-sombre. Trois unités lithostratigraphiques ont été identifiées. L'unité U1 est caractérisée par la prédominance du faciès 1 et dans laquelle s'intercalent trois niveaux plus argileux (faciès 2). Le sédiment de l'unité U2 est homogène et composé d'argiles brunsombre (faciès 3). Le sédiment de l'unité U3 est une diatomite plus ou moins argileuse (faciès 2 ) présentant des lamines diffuses. Au sein de l'unité U3, la sous-unité U3a est relativement plus claire (jaune/brun-clair) qu'en U3b. Dans la sous-unité U3c le faciès 2 est dominant, à exception, à $29 \mathrm{~cm}$ de profondeur, d'une lamine diffuse brun-sombre argileuse de $5 \mathrm{~mm}$ d'épaisseur (faciès 3 ). L'unité U3c est laminée de façon diffuse sauf entre 5 et $0 \mathrm{~cm}$ où le sédiment est homogène. L'analyse granulométrique (fig. 3) reflète une homogénéité du sédiment avec des distributions unimodales et asymétriques vers les petites tailles supposant une source sédimentaire constante sur l'ensemble de la carotte. Le mode granulométrique se situe à $25 \mu \mathrm{m}$, taille caractéristique des diatomées observées à la loupe binoculaire. La quantité de silice biogénique moyenne est de $67 \%$. Tous les échantillons présentent de très faibles proportions en argile avec en moyenne $2 \%$ du volume occupé dans le faciès $1,2,5 \%$ dans le faciès 2 , et $3 \%$ dans le faciès 3 . Le sédiment est globalement riche en matière organique (\%COT compris entre 3 et $15 \%$ ).

\section{3 - ORIGINE DE LA MATIÈRE ORGANIQUE}

Selon un diagramme de type van Krevelen modifié, indice d'hydrogène (IH) vs. indice d'oxygène (IO) (fig. 4), la matière organique apparait de type II prédominante (i.e. d'origine algale vraisemblable; Espitalié et al., 1985) et ce, dans tous les faciès sédimentaires. Il est cependant possible qu'une contribution terrestre puisse être diluée dans la sédimentation autochtone. Le faciès 1 est caractérisé par les indices d'hydrogène les plus élevés $(670 \mathrm{mg}$ $\mathrm{HC} / \mathrm{g} \mathrm{COT})$; il reflète la présence de matière organique très faiblement évoluée produite par le phytoplancton et bien préservée après sédimentation. Les faciès 2 et 3 se distinguent par un IH plus bas autour de $50 \mathrm{mg} \mathrm{HC} / \mathrm{g}$ COT (fig. 4). La diminution de l'IH entre les faciès 1 et 2-3 peut a priori s'expliquer par une augmentation de la part de matière organique terrestre (signal mixte des types algal et plante vasculaire) et/ou par une accentuation de la dégradation de la matière organique. Cette dernière hypothèse est la moins probable du fait de l'excellente préservation de la matière organique (\%COT moyen de $9 \%)$.

\section{4 - SIGNATURES DÉTRITIQUES ET PÉDOLO- GIQUES}

La comparaison des observations lithostratigraphiques ainsi que des analyses des constituants chimiques (organiques et inorganiques) permet de distinguer plusieurs phases de sédimentation.

- L'unité U1 $(144$ à $112 \mathrm{~cm})$ : les profils de $\mathrm{K}$ et Ti ont des valeurs proches de zéro. Il n'y a pas d'apport détritique. Le \%COT est élevé (10\% en moyenne). Les valeurs particulièrement élevées de l'IH suggèrent que la fraction organique est d'origine lacustre prépondérante, voire exclusive. L'abondance élevée en BSi (68 $\%$ montre que les diatomées sont la principale source de sédimentation dans le lac.

- L'unité U2 (112 à $106 \mathrm{~cm}$ ): les deux éléments K et Ti, traceurs du détritisme, enregistrent une brutale augmentation avec des valeurs maximales à $110 \mathrm{~cm}$ égales à 4 fois les valeurs de l'unité U1. Les valeurs de l'IH, plus faible que dans l'unité U1, pourraient signaler soit une altération plus importante de la matière organique, soit une contribution de matière organique terrestre. L'association de faibles valeurs d'IH à un fort détritisme alors que les valeurs en \%COT se maintiennent à un niveau élevé $(12 \%)$ nous amènent à privilégier cette seconde hypothèse. Une modification rapide des conditions de sédimentation se produit donc, caractérisée par une augmentation des apports détritiques venant diluer le sédiment endogène (teneur en BSi de $26 \%$ ).

- L'unité U3 $(106$ à $0 \mathrm{~cm})$ : les profils de Ti et $\mathrm{K}$ redeviennent relativement constants mais l'abondance en matériel détritique est supérieure à ce qui est observé dans l'unité 1 . Le rapport de Ti/Si augmente progressivement de U3a à U3b en parallèle d'une réduction de l'abondance en BSi. Les apports allochtones représentent une part de plus en plus importante de la sédimentation. Ces apports terrigènes s'intensifient dans l'unité U3c.

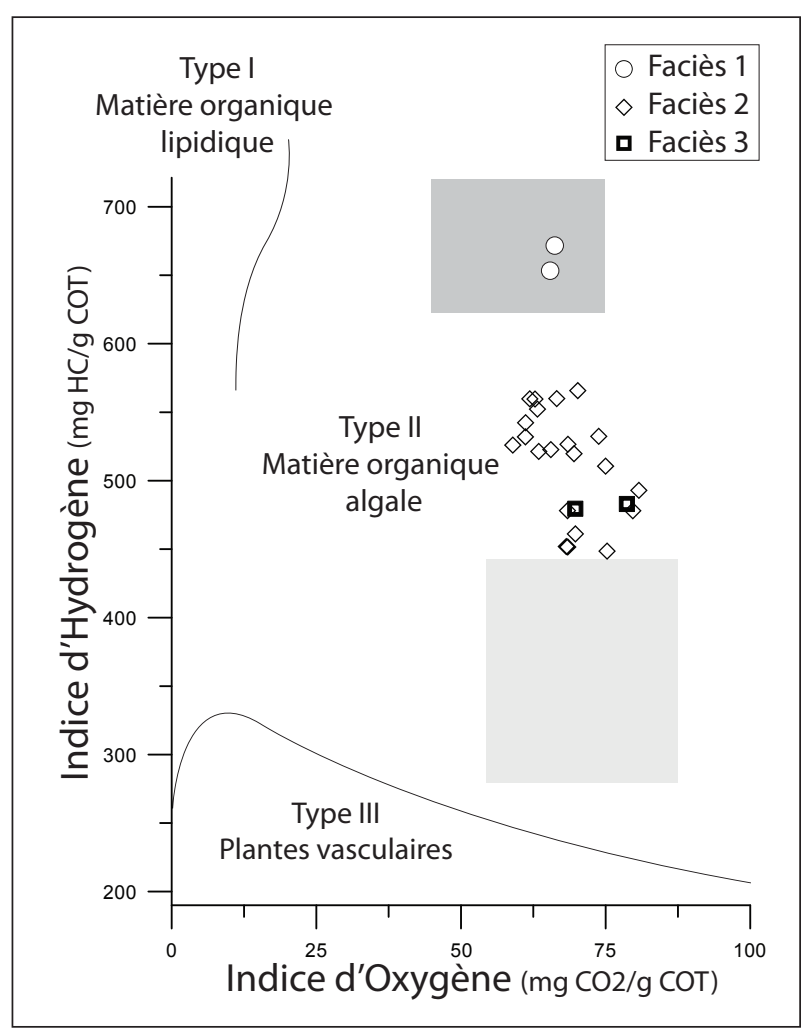

Fig. 4 : Typologie de la matière organique mesurée sur 25 échantillons.

Représentation de type Van Krevelen. La zone en gris foncé indique une signature de type matière organique lacustre et la zone en gris clair signe les horizons pédologiques superficiels (Noël, 2001).

Fig. 4: Organic matter typology measured on 25 samples (Van Krevelen diagram). Dark grey area indicates aquatic organic matter and the lighter one, topmost soil horizons (Noël, 2001). 
Le décalage des profils de $\mathrm{K}$ et Ti vers les valeurs élevées est associé à une variabilité encore plus importante du rapport Ti/Si. Les valeurs de silice biogénique diminuent parallèlement à l'augmentation de la fraction argileuse.

\section{5 - DYNAMIQUE DE LA VÉGÉTATION}

L'enregistrement pollinique du Lac Petit débute par le déclin de la hêtraie-sapinière au profit de la chênaie caducifoliée, comme cela est observé pendant le Subboréal dans les Alpes (de Beaulieu, 1977). Le diagramme est dominé par Pinus (fig. 3). Les fluctuations des autres taxons polliniques sont minorées par les fréquences du pin. Ce taxon est un fort producteur pollinique dont la dissémination par le vent a lieu massivement sur de très longues distances; Pinus est donc souvent surreprésenté dans la pluie pollinique (Coûteaux, 1981), tout particulièrement dans les zones ouvertes de haute montagne. Afin d'améliorer la représentation graphique des autres taxons polliniques, les effectifs de Pinus sont exclus de la somme pollinique totale.

Cependant, les pourcentages polliniques importants et les occurrences régulières de trachéides et/ou de stomates de Pinus témoignent de la présence d'une pinède subalpine à proximité du Lac Petit, au moins dans la première moitié de l'enregistrement. Il reste toutefois difficile d'attester la présence locale d'une pinède dense autour du lac. D'autres études sont prévues (notamment une analyse paléoentomologique) afin de préciser ce point. La question de la présence du mélèze (Larix) autour du lac reste également posée car le pollen de cette espèce est généralement mal dispersé et donc peu ou pas représenté dans la pluie pollinique (de Beaulieu, 1977). Ici, l'absence de pollen de mélèze ne nous permet pas d'exclure sa présence locale, alors qu'il constitue actuellement une essence forestière majeure de l'étage subalpin dans le Mercantour.

La chênaie mixte et la hêtraie-sapinière, également enregistrées dans les sédiments du Lac Petit, étaient probablement localisées plus bas en altitude étant donné leurs exigences écologiques (Ozenda, 2002). Cette étude préliminaire a permis de retracer les grandes étapes de l'évolution des différentes formations forestières (fig. 3).

\subsection{1 - Un environnement forestier (zones polli- niques $P 1$ à $P 4)$ - entre 4750 et 1550 cal. $B P$}

Le début de l'enregistrement pollinique témoigne d'un environnement forestier probablement dense à proximité du site, mais également à plus basse altitude (P1). La hêtraie-sapinière à l'étage montagnard et la chênaie mixte à l'étage collinéen semblent se développer sans perturbation majeure, sauf vers $4260 \mathrm{cal}$. BP (zone pollinique $\mathrm{P} 2$ ): en effet, un évènement probablement rapide (contemporain de l'unité lithostratigraphique et géochimique U2) a significativement perturbé l'ensemble du couvert forestier du vallon de Millefonts comme en témoigne la diminution transitoire du rapport PA/PNA. Jusqu'à la fin de la zone $\mathrm{P} 4$, la chênaie mixte semble se maintenir, alors que la hêtraie-sapinière connaît un déclin progressif (notamment en zone P3). Enfin, même si la courbe de Pinus ne connaît pas de variation significative, la diminution des occurrences de stomates et de trachéides de résineux pourrait également indiquer un recul de la pinède. En l'absence d'étude détaillée des macrorestes végétaux, cette hypothèse ne peut être confirmée.

4.5.2 - Une dégradation générale des paysages de montagne (zone P5) - depuis 1550 cal. BP

La zone P5, contemporaine de la fin du Subatlantique, est caractérisée par la chute brutale du rapport PA/PNA. Les forêts sont fortement affectées à toutes les altitudes par des perturbations d'origine anthropique: en effet, la courbe synthétique des taxons d'anthropisation connaît un optimum contemporain du déclin des forêts. Les pratiques agro-pastorales se sont intensifiées dans l'ensemble du vallon.

\section{5 - UNE HISTOIRE PALÉOENVIRONNEMENTALE EN TROIS PHASES}

La confrontation des données sédimentologiques, géochimiques et botaniques permet de reconstituer trois grandes périodes, au sein desquelles des épisodes détritiques probablement brefs ont contribué à déstabiliser le système (fig. 5).

- Une première période (A), entre 4750 et 4350 cal. BP, est marquée par une relative stabilité du milieu. Un couvert forestier s'étendait vraisemblablement jusqu'aux abords du lac. La sédimentation lacustre est dominante (forte abondance en $\% \mathrm{BSi}$ et pas d'apports terrigènes). Ainsi, les versants et les paysages qu'ils abritent, même s'ils enregistrent déjà les traces de la présence de l'Homme (présence de taxons d'anthropisation), apparaissent en équilibre relatif.

- Une seconde période (B), entre 4180 et 1550 cal. $B P$, est marquée par une première déstabilisation du système. La forêt a probablement subi une première perturbation à proximité du lac (diminution des pourcentages polliniques des taxons arboréens). L'augmentation $\mathrm{du}$ rapport $\mathrm{Ti} / \mathrm{Si}$ indique que les processus érosifs sont épisodiquement activés alors que les sols ne sont plus protégés par la couverture arborée. Le ruissellement des eaux transfère également des particules organiques issues de l'érosion des sols vers le lac comme l'attestent de plus faibles valeurs de l'indice d'hydrogène. Les stomates et trachéides de résineux, principalement apportés par le ruissellement sur les versants, sont de moins en moins fréquents et attestent de la disparition des arbres en amont du lac. Ces apports terrigènes diluent la sédimentation autochtone toujours dominée par les diatomées. Le milieu est sensible aux pulsations érosives.

- Une dernière période $(C)$, postérieure à 1550 cal. $B P$, est marquée par une déstabilisation durable de 


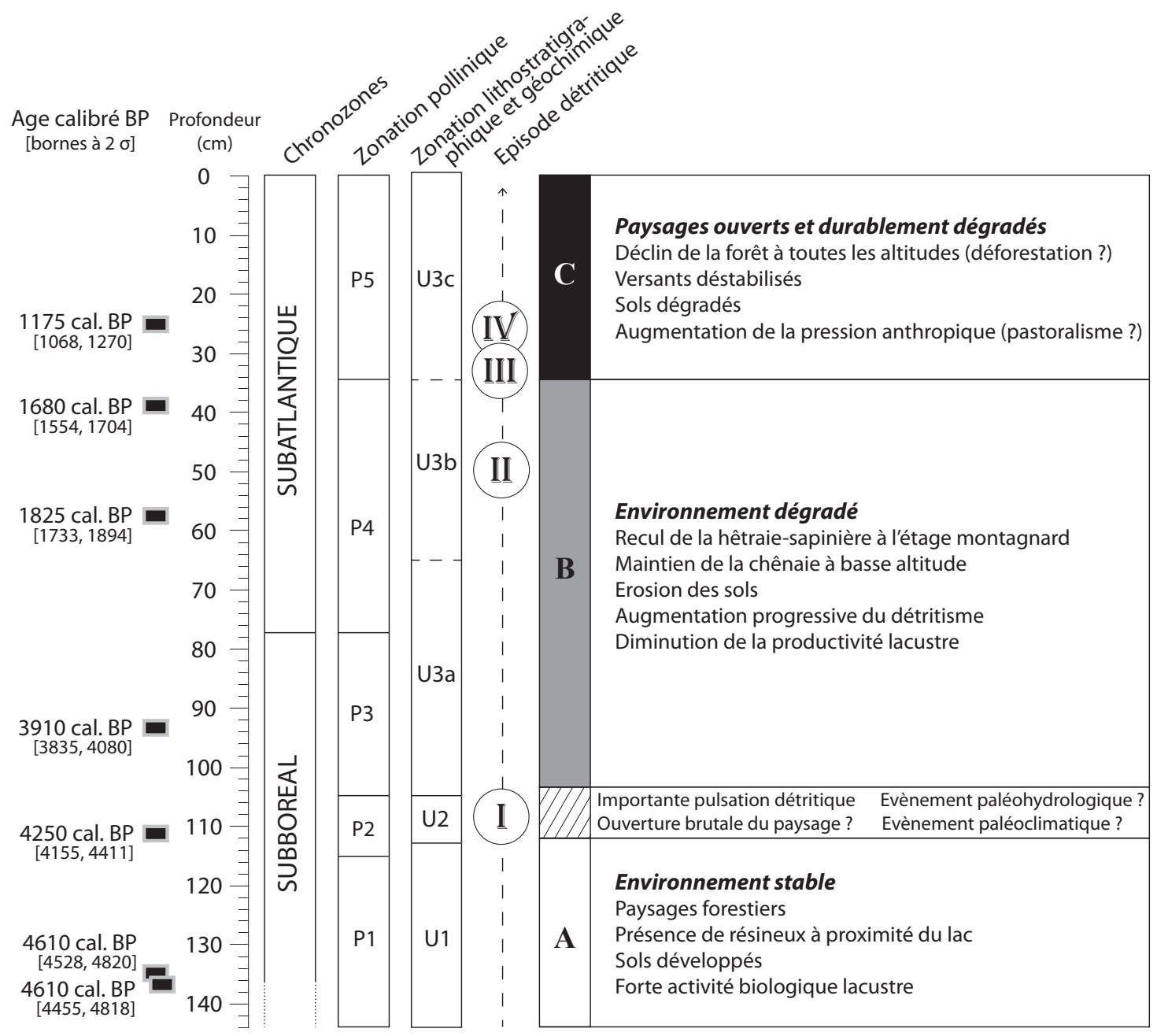

Fig. 5 : Synthèse des principales étapes de la dynamique du système bassin-versant / lac dans le vallon de Millefonts.

Fig. 5: Summary of main palaeoenvironmental changes recorded at Millefonts.

l'environnement. Les données polliniques confirment qu'à toutes les altitudes, le couvert forestier est durablement dégradé : la chute drastique du rapport PA/PNA est accompagnée par la forte recrudescence des taxons d'anthropisation (Plantago, Mentha, Rumex, Urtica, et des Chenopodiaceae). Les apports terrigènes augmentent (fort excès $\mathrm{du} \mathrm{Ti} / \mathrm{Si}$ ) et diluent fortement le signal lacustre (faible $\% \mathrm{BSi}$ ). Au cours de cette période d'intense ouverture des milieux, l'absence de couverture arborée a vraisemblablement rendu les sols vulnérables au ruissellement. Les versants apparaissent durablement sensibles à l'érosion des sols.

$\mathrm{Au}$ sein de ces périodes, l'observation de la courbe $\mathrm{Ti} / \mathrm{Si}$ permet d'identifier quatre pulsations détritiques (I, II, III, IV, fig. 3 et 5).

La pulsation I (4350 - 4180 cal. BP), marquée par un maximum des courbes de $\mathrm{Ti} / \mathrm{Si}, \mathrm{K}$ et $\mathrm{Ti}$, témoigne d'une perturbation majeure des versants: un pic de détritisme s'accompagne du déclin transitoire du couvert forestier au profit des herbacées.

Les pulsations II, III et IV (respectivement datées à 1710 cal. BP, 1450 cal. BP et 1260 cal. BP) sont caractérisées par d'importants excès en $\mathrm{Ti} / \mathrm{Si}$, sans que les abondances relatives en $\mathrm{K}$ et $\mathrm{Ti}$ ne soient élevées. Ces évènements sont portés par une chute de l'abondance en silice biogénique, laquelle est en partie diluée par les apports terrigènes.

\section{6 - DISCUSSION}

\section{1 - UNE SÉDIMENTATION SOUS CONTRÔLE CLIMATIQUE?}

Des fluctuations climatiques identifiées pendant l'Holocène peuvent-elles expliquer pour partie les changements de sédimentation observés dans le Lac Petit? Dans le vallon de Millefonts, le passage de versants stabilisés (Phase A, fig. 5) à des versants déstabilisés (Phase B puis C) autour de 4350 cal. BP pourrait être attribué à un changement de régime climatique, identifié entre l'Holocène moyen et final. En effet, dans les Alpes Suisses, les reconstitutions climatiques basées sur des assemblages de chironomes (Larocque-Tobler et al., 2010) indiquent une diminution des températures d'été à partir de $4900 \mathrm{cal}$. BP. Aux lacs Accesa et Cerin en Italie du Nord et dans les Alpes, Magny et al. (2011) mettent en évidence un changement de saisonnalité, avec des maxima de précipitation en 
hiver entre 10000 et 4500 cal. BP et un basculement vers des maxima de précipitations d'été depuis $4500 \mathrm{cal}$. BP. Une augmentation des volumes de précipitation liquide en saison estivale a pu accroître localement les apports détritiques dans le Lac Petit, pendant qu'une chute des températures a pu provoquer un recul de la limite supérieure des forêts et de la couverture pédologique associée. Dans le Valais suisse, Finsinger \& Tinner (2007) montrent un recul de la limite supérieure de la forêt dès $4700 \mathrm{cal}$. BP au début du Néoglaciaire, précédant les déforestations massives d'origine anthropique débutant vers $3500 \mathrm{cal}$. BP. L'expression de cette transition climatique apparaît comme un changement d'hydrodynamisme et de source des processus érosifs dans l'enregistrement du Lac d'Anterne (Alpes françaises du Nord), avec une augmentation de l'érosion des sols (aussi bien des horizons superficiels que de subsurface) ainsi que de la fréquence des crues entre 5500 et 4600 cal. BP (Giguet-Covex et al., 2011). Dans la haute vallée du Rhône également, à 4300 cal. BP, cette période est caractérisée par une augmentation des phases d'activité hydro-sédimentaire intenses des grands et petits bassin-versants (Berger et al., 2008). Enfin, dans la vallée de la Maurienne en Savoie, l'enregistrement sédimentaire du Loup (Mourier, 2008; Mourier et al., 2010) montre qu'entre 4500 et $1100 \mathrm{cal}$. BP, les processus de podzolisation et la productivité biogénique lacustre diminuent notablement. En l'absence d'indice de déstabilisation lié à des activités anthropiques, Mourier (2008) interprète cette diminution de la sédimentation lacustre et des sols comme liée à une baisse des températures. En revanche, à Millefonts, la baisse de la productivité lacustre à partir de 4350 cal. BP s'accompagne d'une augmentation de l'érosion des sols et d'une ouverture marquée des paysages. Le système ne se stabilise pas avec le temps et semble au contraire de plus en plus vulnérable.

\section{2 - UNE PRESSION ANTHROPIQUE EXCESSIVE DANS LE VALLON DE MILLEFONTS?}

Dans les Alpes, il ne fait aucun doute que le forçage anthropique se superpose - voire masque - le signal climatique dans tout enregistrement sédimentaire, au moins depuis l'âge du Bronze. Même si aucun site d'occupation n'a été jusqu'à présent mis en évidence dans le vallon de Millefonts pour cette époque (Suméra et al., 2008), la distribution et le nombre de sites recensés dans les Alpes du Sud (Ubaye, massif des Ecrins, Mocci et al. 2008a) montrent une hausse de la fréquentation de la haute montagne. L'analyse pollinique du Lac Petit témoigne de la présence d'un cortège floristique «anthropogénique» dès la base de la séquence (fig. 3) certes discret mais représentant une part significative du pollen non arboréen. Le vallon a pu accueillir des activités agro-pastorales avant 4750 cal. BP d'abord discrètes, puis de manière intensive lorsque les courbes des taxons herbacés "anthropiques» et de Juglans augmentent (zone P4, phase C, fig. 3 et 5). Les occurrences de noyer indiquent que cette anthropisation massive est contemporaine de l'époque romaine. L'optimum de Juglans (milieu de la zone $\mathrm{P} 5$, fig. 3) à environ $500 \mathrm{cal}$. BP pourrait être contemporain du Moyen Âge, période pour laquelle plusieurs enclos à vocation pastorale ont été identifiés dans le bassin-versant (Suméra, 2007). Pendant cette période, les sols et le couvert forestier sont particulièrement dégradés, et ce probablement sur l'ensemble du vallon de Millefonts (phase C, fig. 5).

L'extension progressive des aires de pâturage semble donc avoir contribué à déstabiliser durablement la couverture pédologique et les écosystèmes du vallon, même si la variabilité climatique holocène a pu jouer un rôle dans l'ouverture des paysages. Il faut probablement considérer qu'une combinaison complexe des forçages climatiques et anthropiques est responsable des grandes phases de changements des paysages dans le vallon de Millefonts et les pulsations détritiques enregistrées au Lac Petit en sont l'expression.

\section{3 - DES PULSATIONS DÉTRITIQUES «RAPIDES » QUELS DÉCLENCHEURS?}

L'épisode I (fig. 3 et 5) daté entre 4350 - 4180 cal. BP, est probablement le plus important qu'ait connu le Lac Petit sur la période étudiée. Cet épisode ne semble pas apparaître dans les autres enregistrements polliniques régionaux, sauf au Lac des Grenouilles (Commune de Tende). Dans ce site, Kharbouch (2000) reconstitue un enregistrement pollinique comparable à celui du Lac Petit, marqué par la contraction brutale des taxons arboréens (palyno-stratigraphiquement datée du milieu du Subboréal postérieurement à l'optimum d'Abies Zone $\mathrm{H}$ ). L'auteur réfute l'hypothèse climatique considérant que la chute concomitante de presque tous les taxons arboréens ne refléterait pas une dynamique régressive naturelle de la végétation. Des évènements détritiques dans les fonds de vallon alluviaux sont également identifiés à 4410 - 4090 cal. BP dans les Alpes du Sud (Rousset \& Guiomar, 1999), entre 4450 et 3700 cal. BP dans l'enregistrement des crues du Rhône au Lac du Bourget (Arnaud et al., 2005) ainsi que dans la plaine alluviale du Rhône entre 4250 et 3880 cal. BP (Delhon, 2005; Berger et al., 2008). Dans les Alpes du Sud, le Lac de Saint Léger connaît un haut niveau entre 5200 et 3700 cal. BP (Digerfeldt et al., 1997). Au même moment entre 4150 et $3950 \mathrm{cal}$. BP, des hauts niveaux lacustres sont enregistrés dans les Alpes du Nord et dans le Jura (Magny, 2004). L'extension à l'échelle européenne de cet évènement dans des archives sédimentaires variées d'Europe de l'Ouest mais aussi dans le bassin méditerranéen (Magny et al., 2011), suggère l'occurrence d'une importante anomalie climatique à $4200 \mathrm{cal}$. BP dont les forçages sont encore discutés (Magny et al., 2009).

Les épisodes II (1710 cal. BP) et III (1450 cal. BP) interviennent dans un contexte où le déboisement du bassin a probablement débuté avec pour conséquence une déstabilisation des versants. Morin et Rosenthal (2003) ont daté des résidus d'extraction de minerais entre 2000 et 1500 cal. BP, c'est-à-dire possiblement contemporains de ces pulsations détritiques. Des activités minières ont pu accentuer la déstabilisation des versants; les épisodes II et III pourraient représenter alors des crises rapides, 
liées à une surexploitation des ressources (minerai et combustible ligneux). Le rôle du climat ne doit pas être pour autant écarté. Ces événements détritiques, de durée mal connue, peuvent refléter des fluctuations régionales du climat, mais aussi des évènements orageux brefs qui auraient activé un ruissellement généralisé sur des versants déjà dégradés par les activités humaines.

L'épisode IV (1260 cal. BP) intervient en même temps que la chute brutale et définitive de la couverture forestière à l'étage subalpin. Ici, tout porte à croire que la recrudescence de l'érosion des sols est directement en relation avec les exploitations agro-pastorales, forestières, voire minières du bassin-versant.

\section{7 - CONCLUSION ET PERSPECTIVES}

Le travail présenté ici est la première étude multidisciplinaire associant des analyses sédimentologiques, géochimiques et paléoécologiques menée dans les Alpes du Sud. L'approche sédimentologique a permis de reconstituer le fonctionnement original d'un petit lac de montagne, non conditionné par des apports détritiques saisonniers mais dominé par une sédimentation diatomitique lacustre relativement continue. Nous avons montré que la principale source en matériel terrigène est assurée par la dégradation des sols adjacents au lac. Le croisement des données sédimentologiques, géochimiques et palynologiques a montré que des phases de stabilité relative et de déstabilisation du milieu naturel se sont succédé au moment où les Hommes défrichent et exploitent un milieu de montagne probablement déjà fragile. Des analyses polliniques à haute résolution et de nouvelles datations radiocarbone sont en cours. Une analyse paléoentomologique est prévue afin de compléter la reconstitution paléoécologique autour du lac. D'autres analyses géochimiques de dosage élémentaire sur échantillons ponctuels sont envisagées pour discuter de l'impact des activités de réduction de minerai attestées par l'archéologie (Morin \& Rosenthal, 2003; Pagès, 2009) sur l'évolution paléoenvironnementale du bassin-versant. Par ailleurs, l'extraordinaire abondance en diatomées offre la perspective de reconstructions paléohydrologiques. La comparaison avec d'autres sites régionaux permettra de discriminer la part respective des composantes climatiques et anthropiques. Enfin, une nouvelle opération de carottage est envisagée pour étudier la base du remplissage lacustre, c'est-à-dire la première partie de l'Holocène durant laquelle les impacts de l'Homme sont peu présents. Il serait ainsi possible de calibrer les signaux naturels avant que les environnements ne soient soumis à l'impact de la pression anthropique.

\section{REMERCIEMENTS}

Les auteurs remercient chaleureusement Kazuyo Tachikawa et Marta Garcia (CEREGE) pour la mise à disposition de l'Itrax Core Scanner et de leur aide lors de l'analyse de la carotte. Un grand merci à Emmanuel Malet (EDYTEM) pour son aide précieuse dans le prélèvement de la carotte ainsi qu'à l'association A.M.O.N.T. (Association Montagne et Traditions, Saint Martin de Vésubie) pour leur participation lors du carottage. Nous remercions également les relecteurs T. Adatte, M. Magny et un relecteur anonyme pour leur aide à l'amélioration du manuscrit.

\section{RÉFÉRENCES BIBLIOGRAPHIQUES}

ANCEL B., 2010 - Les anciennes mines métalliques des Alpes du Sud : bilan diachronique. Bibliothèque d'Archéologie Méditerranéenne et Africaine, 4, 285-292.

ARNAUD F., REVEL-ROLLAND M., CHAPRON E., DESMET M. \& TRIBOVILLARD N., 2005 - 7200 years of Rhône river flooding activity recorded in Lake Le Bourget: A high resolution sediment record of NW Alps hydrology. The Holocene, 15 (3), 420-428.

BERGER J.-F., SILVADOR P.-G. \& FRANC O., 2008 - La chronologie fluviale postglaciaire du haut bassin rhodanien. Collection EDYTEM. Cahiers de Paléoenvironnement, 6, 117-144.

BOURGARIT D., ROSTAN P., BURGER E., CAROZZA L., MILLE B. \& ARTIOLI G., 2008 - The beginning of copper mass production in the western Alps: the Saint-Véran mining area reconsidered. Historical Metallurgy, 42 (1), 1-11.

COURT-PICON M., BUTTLER A. \& DE BEAULIEU J.-L., 2005

- Modern pollen-vegetation relationships in the Champsaur valley (French Alps) and their potential in the interpretation of fossil pollen records of past cultural landscapes. Review of Palaeobotany and Palynology, 135 (1/2), 13-39.

COÛTEAUX M., 1982 - Caractérisation pollenanalytique en Oisans des milieux forestiers et des milieux supraforestiers dans l'actuel et dans le passé. In G. Soutadé, A. Baudière \& J. Bécat (eds.), La limite supérieure de la forêt et sa valeur de seuil: Colloque de Perpignan, 16 avril 1981. Institut d'Estudis Andorrans, Perpignan, 139-159.

CROUDACE I.W., RINDBY A. \& ROTHWELL R.G., 2006 ITRAX: description and evaluation of a new multi-function X-ray core scanner. Geological Society Special Publication, 267, 51-63.

DE BEAULIEU J.-L., 1977 - Contribution pollenanalytique à l'histoire tardiglaciaire et holocène de la végétation des Alpes méridionales françaises. Thèse de Doctorat, Université Paul Cézanne, Aix-Marseille, $358 \mathrm{p}$.

DELHON C., 2005 - Anthropisation et paléoclimats du Tardiglaciaire à l'Holocène en moyenne vallée du Rhône: études pluridisciplinaires des spectres phytolithiques et pédo-anthracologiques de séquences naturelles et de sites archéologiques. Thèse de Doctorat, Université Paris I Panthéon-Sorbonne, Paris, 3 vol., 843 p.

DEMANGEOT J., 1984 - Les Milieux «naturels » du globe. Masson, Paris, $250 \mathrm{p}$.

DEMASTER D.J., 1981 - The supply and accumulation of silica in the marine environment. Geochimica et Cosmochimica Acta, 45 (10), 1715-1732.

DIGERFELDT G., BEAULIEU de J.-L., GUIOT J. \& MOUTHON J., 1997 - Reconstruction and paleoclimatic interpretation of Holocene lake-level changes in Lac de Saint Léger, Haute-Provence, southeast France. Palaeogeography, Palaeoclimatology, Palaeoeco$\log y, 136(1 / 4), 231-258$.

ESPITALIÉ J., DEROO G. \& MARQUIS F., 1985 - La pyrolyse Rock-Eval et ses applications. Oil \& Gas Science and Technology, 40 (5), 563-579.

FAEGRI K. \& IVERSEN J., 1989 - Textbook of Pollen Analysis. $4^{e}$ édition, Wiley \& Sons, New York, 328 p.

FINSINGER W. \&TINNER W., 2007 - Pollen and plant macrofossils at Lac de Fully (2135 $\mathrm{m}$ a.s.1.): Holocene forest dynamics on a highland plateau in the Valais, Switzerland. The Holocene, 17 (8), 11-19.

GARCIA D., MOCCI F., TZORTZIS S. \& WALSH K., avec la collaboration de DUMAS V., 2007 - Archéologie de la vallée de l'Ubaye (Alpes-de-Hautes-Provence, France): présentation des premiers résultats d'un Projet Collectif de Recherche. Prehistoria Alpina, 42, 23-48.

GIGUET-COVEX, C., ARNAUD F., POULENARD J., DISNAR J.-R., DELHON C., FRANCUS P., DAVID F., ENTERS D., REY P.-J. \& DELANNOY J.-J., 2011 - Changes in erosion patterns during the Holocene in a currently treeless subalpine catchment inferred from lake sediment geochemistry (Lake Anterne, $2063 \mathrm{~m}$ a.s.l., NW French Alps): The role of climate and human activities. The Holocene, 21 (4), 651-665. 
HIGUERA P.E., BRUBAKER L.B., ANDERSON P.M., SHENG HU F. \& BROWN T.A., 2009 - Vegetation mediated the impacts of postglacial climate change on fire regimes in the south-central Brooks Range, Alaska. Ecological Monographs, 72 (2), 201-219.

KHARBOUCH M., 2000 - L'homme et la végétation dans la région du mont Bego (Tende, Alpes-Maritimes) depuis des millénaires. Comptes Rendus de l'Académie des Sciences. Série 2. Sciences de la Terre et des Planètes, 330 (12), 889-894.

LAROCQUE-TOBLER I., HEIRI O. \& WEHRLI M., 2010 - Late Glacial and Holocene temperature changes at Egelsee, Switzerland, reconstructed using subfossil chironomids. Journal of Paleolimno$\log y, 43$ (4), 649-666.

MAGNY M., 2004 - Holocene climate variability as reflected by midEuropean lake-level fluctuations and its probable impact on prehistoric human settlements. Quaternary International, 113 (1), 65-79.

MAGNY M., VANNIERE B., ZANCHETTA G., FOUACHE E., TOUCHAIS G., PETRIKA L., COUSSOT C., WALTERSIMONNET A.-V. \& ARNAUD F., 2009 - Possible complexity of the climatic event around 4300-3800 cal. BP in the central and western Mediterranean. The Holocene, 19 (6), 823-833.

MAGNY M., VANNIẼRE B., CALO C., MILLET L., LEROUXA. PEYRON O., ZANCHETTA G., LA MANTIA T. \& TINNER W., 2011 - Holocene hydrological changes in south-western Mediterranean as recorded by lake-level fluctuations at Lago Preola, a coastal lake in southern Sicily, Italy. Ouaternary Science Reviews, $30(19 / 20), 2459-2475$

MOCCI F., TZORTZIS S., PALET-MARTINEZ J., SEGARD M. \& WALSH K., 2005 - Peuplement, pastoralisme et modes d'exploitation de la moyenne et haute montagne depuis la Préhistoire dans le Parc national des Ecrins (vallée du haut Champsaur et de Freissinières, Hautes-Alpes). In A. Bouet \& F. Verdin (eds.), Territoires et paysages de l'âge du fer au Moyen Âge: Mélanges offerts à Philippe Leveau. Ausonius, Bordeaux, 197-212.

MOCCI F., WALSH K., RICHER S., COURT-PICON M., TALON B., TZORTZIS S., PALET-MARTINEZ J. \& BRESSY C. 2008a - Archéologie et paléoenvironnement dans les Alpes méridionales françaises: Hauts massifs de l'argentiérois, du Champsaur et de l'Ubaye, Hautes-Alpes et Alpes-de-Haute-Provence, Néolithique final - début de l'Antiquité. Collection EDYTEM. Cahiers de Paléoenvironnement, 6, 253-272.

MOCCI F., WALSH K., TALON B., TZORTZIS S. \& COURTPICON M., 2008b - Structures pastorales d'altitude et paléoenvironnement. In J.-P. Jospin \& T. Favrie (dir.), Premiers bergers des Alpes de la préhistoire à l'Antiquité. Infolio, Gollion, 92-101.

MORIN D. \& ROSENTHAL P., 2003 - Mines et métallurgie du fer en Provence et dans les Alpes du Sud. Saint-Dalmas, Valdeblore. Etude diachronique des vestiges d'exploitation minière et de métallurgie du fer, prospection au sol. Document final de synthèse, Service Régional d'Archéologie de Provence-Alpes-Côte-d'Azur, Aix-enProvence, $19 \mathrm{p}$.

MOURIER B., 2008 - Contribution de l'approche sédimentologique à la reconstitution de l'histoire des sols. Définitions de traceurs pédologiques et application sur des sédiments lacustres. Thèse de Doctorat, Université de Savoie, Chambéry, 214 p.
MOURIER B., POULENARD J., CARCAILLET C. \& WILLIAMSON D., 2010 - Soil evolution and subalpine ecosystem changes in the French Alps inferred from geochemical analysis of lacustrine sediments. Journal of Paleolimnology, 44 (2), 571-587.

NICOD P.-Y., 2008 - Les premières sociétés agropastorales dans les Alpes occidentales. In J.-P. Jospin \& T. Favrie (dir.), Premiers bergers des Alpes : de la Préhistoire à l'Antiquité. Infolio, Gollion, 45-51.

NOËL H., 2001 - Caractérisation et calibration des flux organiques sédimentaires dérivant du bassin versant et de la production aquatique (Annecy, Le Petit Lac). Rôles respectifs de l'Homme et du Climat sur l'évolution des flux organiques au cours des 6000 dernières années. Thèse de Doctorat, Université d'Orléans, Orléans, 272 p.

OZENDA P., 2002 - Perspectives pour une géobiologie des montagnes. Presses Polytechniques et Universitaires Romandes, Lausanne, 208 p.

PAGÈS G., 2009 - Le Clouté Valdeblore, rapport de fouilles programmées. Rapport de fouilles programmées, Service Régional d'Archéologie de Provence-Alpes-Côte-d'Azur, Aix en Provence, 42 p.

REIMER P.J., BAILLIE M.G.L., BARD E., BAYLISS A., BECK J.W., BLACKWELL P.G., BRONK RAMSEY C., BUCK C.E. BURR G.S., EDWARDS R.L., FRIEDRICH M., GROOTES P.M., GUILDERSON T.P., HAJDAS I., HEATON T.J., HOGG A.G., HUGHEN K.A., KAISER K.F., KROMER B., MC CORMAC F.G., MANNING S.W., REIMER R.W., RICHARDS D.A., SOUTHON J.R., TALAMO S., TURNEY C.S.M., VAN DER PLICHT J. \& WEYHENMEYER C.E., 2009 - IntCal09 and Marine09 Radiocarbon Age Calibration Curves, 0-50,000 Years cal BP. Radiocarbon, 51 (4), 1111-1150.

ROSÉN P., VOGEL H., CUNNINGHAM L., REUSS N., CONLEY D. \& PERSSON P., 2010 - Fourier transform infrared spectroscopy, a new method for rapid determination of total organic and inorganic carbon and biogenic silica concentration in lake sediments. Journal of Paleolimnology, 43 (2), 247-259.

ROSTAN P. \& ROSSI P., 2002 - Approche économique et industrielle du complexe minier et métallurgique de Saint-Véran (Hautes-Alpes) dans le contexte de l'Age du Bronze dans les Alpes du sud. Bulletin d'Etudes Préhistoriques et Archéologiques Alpines, 13, 77-96.

ROUSSET C. \& GUIOMAR M., 1999 - Le début de la torrentialité dans le bassin versant de la Fontarasse, près de Barles (Alpes-deHaute-Provence, France), marqueur d'une rupture climatique de l'Holocène ancien. Comptes Rendus de l'Académie des Sciences. Série 2. Sciences de la Terre et des Planètes, 328 (8), 541-546.

SUMÉRA F, 2007 - Rapport de prospection et de fouilles, 2004, vallon de Millefonts, Valdeblore, Alpes-Maritimes. Rapport de prospection et de fouilles, Service Régional d'Archéologie de Provence-AlpesCôte-d'Azur, Aix en Provence, 130 p.

SUMÉRA F., BLANC F., CAVANA E., CHOLET V., DELHON C. GAUTIER M. \& MARTIN L., 2008 - Rapport de sondage, commune de Valdeblore. Rapport final d'opération, Service Régional d'Archéologie de Provence-Alpes-Côte-d'Azur, Aix en Provence, 30 p. 